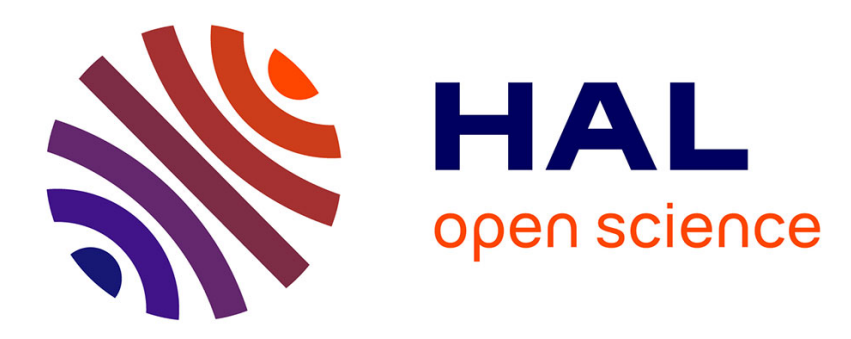

\title{
Alliés de circonstance: Ralph Nader et l'écologie politique
}

Nicolas Labarre

\section{To cite this version:}

Nicolas Labarre. Alliés de circonstance: Ralph Nader et l'écologie politique. Écologie \& politique: sciences, culture, société, 2011, 41, pp.25-38. 10.3917/ecopo.041.0025 . hal-01122639

\section{HAL Id: hal-01122639 \\ https://hal.science/hal-01122639}

Submitted on 4 Mar 2015

HAL is a multi-disciplinary open access archive for the deposit and dissemination of scientific research documents, whether they are published or not. The documents may come from teaching and research institutions in France or abroad, or from public or private research centers.
L'archive ouverte pluridisciplinaire HAL, est destinée au dépôt et à la diffusion de documents scientifiques de niveau recherche, publiés ou non, émanant des établissements d'enseignement et de recherche français ou étrangers, des laboratoires publics ou privés. 


\title{
Alliés de circonstance : Ralph Nader et l'écologie politique
}

\author{
NiCOLAS LABARRE*
}

Les élections présidentielles aux États-Unis ont été marquées, de 1996 à 2008, par la présence de candidats écologistes, dont l'influence a culminé en 2000. Ce développement d'une écologie politique à l'échelle fédérale, désormais capable de faire basculer un scrutin, se fait dans l'ombre du candidat du parti en 1996 et 2000, Ralph Nader. « Icône ${ }^{1}$ » à l'influence considérable sur les politiques publiques dans les années 1960 et 1970, Nader sera le représentant d'un parti auquel il n'adhérera pas, qu'il tiendra à distance mais auquel il parviendra à donner une visibilité inédite. C'est de cette alliance temporaire que découle le moment singulier de l'élection de 2000, qui a pu faire écrire au très conservateur Wall Street Journal: «L'Amérique n'a jamais pris au sérieux un candidat "vert" auparavant, et elle ne le fera plus jamais ${ }^{2}$.» La stratégie de l'homme providentiel, du candidat iconique, était porteuse de promesses, en partie tenues, mais aussi de risques profonds pour un mouvement écologiste ancré dans l'activisme local, fortement handicapé au moment d'investir un champ électoral national défini par la personnalisation des scrutins.

\section{Écologie militante, écologie politique}

En 1962, l'écrivain et scientifique Rachel Carson inventorie dans Silent spring les dégradations de l'environnement dues à l'agriculture intensive, à l'industrie et au complexe de production énergétique ${ }^{3}$. Sérialisé dans le New Yorker, le livre devient un best-seller et fait entrer l'écologie aux États-Unis dans une ère d'activisme local, autour d'une question redécouverte et repensée ${ }^{4}$, qui succède au dialogue institutionnel et consensuel qui existait depuis la fin du XIX ${ }^{\mathrm{e}}$ siè-

* Nicolas Labarre est maître de conférences en civilisation américaine à l'université de Bordeaux 3. Il travaille sur les questions de légitimité culturelle, sur la bande dessinée et sur la représentation du corps politique dans la culture populaire.

1. J. Martin, Nader, crusader, spoiler, icon, Perseus Publishing, Cambridge, 2002. Sauf indication contraire, les éléments biographiques qui suivent proviennent de cet ouvrage.

2. K. Strassel, «It isn't easy being green. Why the Nader vote collapsed», The Wall Street Journal, 9 novembre 2000.

3. R. Carson, Silent spring, Houghton Mifflin, Boston, 1962 [traduction française: Printemps silencieux, Wildproject, Paris, 2009].

4. Sur l'affaiblissement de l'anthropocentrisme à partir de Silent spring, voir L. H. Sideris et K. D. Moore, Rachel Carson. Legacy and challenge, State Univ. of New York Press, Albany, 2008, p. 94-96. La date de 1962 ne fait pas l'unanimité chez les chercheurs, certains préférant prendre pour origine de la prise de conscience environnementale l'année 1970, durant laquelle fut organisé le premier «Earth Day » (la «journée de la terre»). Voir S. P. Hays, Beauty health and permanence. Environmental politics in the USA, 1955-1985, Cambridge Univ. Press, Cambridge, 1987, p. 52-53. 
cle entre des organisations environnementalistes comme le Sierra Club (créé en 1892) et le gouvernement fédéral. La fin des années 1960 et le début des années 1970 sont ainsi marqués par l'apparition de nombreux groupes comme Friends of the Earth en 1969 ou Greenpeace en 1971, tandis que les organisations antérieures comme le Sierra Club sont réactivées. Le nombre de personnes affiliées à des groupes investis dans les questions environnementales passe de 124000 en 1960 à 819000 en 1969, pour frôler les deux millions en $1983^{5}$. Si la création du National Endowment for the Arts, en 1963, avait marqué l'entrée de la culture dans le champ du gouvernement fédéral, celle de l'Environmental Protection Agency (EPA), en 1970, consacre la place nouvelle de l'écologie. Entre 1963 et 1980, le Congrès adopte également un ensemble de lois destinées à lutter contre les multiples formes de pollution, par l'instauration de normes sur les émissions de monoxyde de carbone, la protection de la faune ou la qualité de l'eau potable. La trajectoire de Ralph Nader, lobbyiste défenseur des consommateurs, croise alors celle des mouvements écologistes, puisque certains des groupes de pression qu'il a formés participent à l'élaboration de ces éléments de réglementation.

La présidence de Ronald Reagan met fin à ce relatif consensus ${ }^{6}$, avec notamment le renouvellement de la direction de l'EPA, le relâchement des normes environnementales et l'ouverture à l'exploitation de zones jusque-là protégées ${ }^{7}$. George T. Frampton Jr., président de l'organisation The Wilderness Society, parlera de «dommages irréparables» causés durant les deux mandats de Reagan ${ }^{8}$. L'écologie devient un enjeu de débat politique direct, comme l'illustre en 1982 un rapport très médiatisé, préparé par dix organisations environnementales, en forme de mise en accusation de la politique menée par l'administration Reagan ${ }^{9}$. En 1984, se forme le Green Comittees of Correspondence, coordination lâche de partis et groupes locaux, inspirée par les succès des partis européens. Dix valeurs fondamentales («key values»), toujours centrales aujourd'hui, sont alors avancées comme fondement idéologique de l'organisation: sagesse écologique, démocratie locale («grassroots democracy»), promotion d'une économie localisée et équitable, décentralisation, non-violence, justice sociale, féminisme, respect de la diversité, implication personnelle et globale, attention portée au futur et à la durabilité.

Des sondages réalisés en 1989 et 1990 montrent ainsi que trois Américains sur quatre se disent «environnementalistes»; le Parti républicain a alors logiquement perdu la confiance de ses propres électeurs sur la question. En retour, les démocrates se font les champions de la question, et le clivage entre les deux

5. M. Schreuers, Environmental politics in Japan, Germany and the USA, Cambridge Univ. Press, Cambridge, 2002, p. 32-35 et p. 65-68.

6. Une étude des votes des membres du Congrès sur les questions environnementales montre en effet que le clivage entre démocrates (plutôt favorables à ces législations) et républicains (plutôt défavorables) s'était creusé au cours des années 1970 et allait continuer à s'amplifier jusque dans les années 1990. S. Kramieneski, «Political parties and environmental policy», in J. Lester (dir), Environmental politics and policy, Duke Univ. Press, Durham, 1995, p. 156.

7. R. Dunlap, «Public opinion and environmental policy», in J. Lester, op. cit., p. 87-88.

8. Cité par P. Shabecoff, «Reagan and environment. To many, a stalemate», The New York Times, 2 janvier 1989.

9. R. Dunlap, op. cit., p. 88. 
partis sur la question environnementale atteint des proportions inconnues jusqu'alors ${ }^{10}$. En 1992, alors que George Bush vient de rejeter l'accord de Rio, le futur vice-président Al Gore écrit Earth in the balance, dans lequel il plaide pour une politique environnementale globale ${ }^{11}$. Ses propositions seront reprises dans le programme du Parti démocrate pour l'élection présidentielle: opposition à tout forage pétrolier en Alaska, intégration des clauses environnementales dans les accords de libre-échange ou encore affirmation de la nécessité de traiter à l'échelle internationale les problèmes du réchauffement climatique, de la biodiversité et de la croissance de la population mondiale ${ }^{12}$. Quasi simultanément, en 1991, apparaît ainsi le premier parti écologique à l'échelle des États-Unis, le Parti vert des États-Unis (Green Party USA ou GPUSA), à la gauche du Parti démocrate, qui prend soin d'affirmer son attachement à la préservation de l'équilibre entre l'action électorale et l'activisme local sur lequel il s'est construit ${ }^{13}$. Absents de l'élection présidentielle de 1992, les écologistes gagnent en quelques années une véritable implantation locale dans l'ouest des États-Unis (menant le Parti démocrate à s'inquiéter de la possibilité de triangulaires qui les pénaliseraient, dès 1994), sans équivalent au niveau fédéral ${ }^{14}$.

David Reynolds, chercheur et activiste, a identifié en 1997 trois obstacles s'opposant à cette émergence au niveau fédéral d'un troisième parti : le système électoral, d'abord, qui conditionne l'accès aux scrutins aux résultats obtenus précédemment, le rôle de l'argent et des médias ensuite, qui se concentrent sur les candidats établis, et la grande personnalisation du système politique enfin ${ }^{15}$. Chacun de ces obstacles est particulièrement handicapant dans le cas d'un parti mettant en avant la démocratie locale, qui ne favorise pas l'émergence d'individus emblématiques. Ainsi, en Californie, c'est un ancien démocrate, très modéré sur les questions écologiques mais populaire, qui est présenté par le parti pour les élections au poste de gouverneur, en 1994. Ainsi encore, c'est à Ralph Nader que les Verts de Californie demandent en 1996 d'être leur candidat à l'élection présidentielle. La notoriété de l'homme et son image publique irréprochable permettent à tout le moins d'espérer contourner le dernier obstacle mentionné par Reynolds.

\section{Ralph Nader, avocat des consommateurs}

Né en 1934, diplômé de Harvard et Princenton, Nader, alors jeune avocat, se fait connaître au milieu des années 1960, en devenant le symbole de la lutte des consommateurs contre les abus des grandes entreprises. Ses pre-

10. Ibid., p. $94-95$ et S. Kramieneski, op. cit., p. 164.

11. A. Gore, Earth in the balance. Forging a new common purpose, Earthscan, Londres, 1992.

12. G. Djahansouz, «L'écologie américaine et son avenir politique», Revue française d'études américaines, $\mathrm{n}^{\circ}$ 70, octobre, 1996, p. 53-61, ainsi que S. Kramieneski, op. cit., p. 153-165 et, sur l'élection de 1992, S. J. Buck, Understanding environmental administration and law, Island Press, Washington, 1996, p. 26-28.

13. D. Reynolds, Democracy unbound. Progressive challenges to the two party system, South End Press, Boston, 1997, p. 246.

14. D. Johnson, «Rebellion of Greens is brewing in the West», The New York Times, 24 juillet 1994.

15. D. Reynolds, op. cit., p. 255-257. 
miers travaux portent sur les pratiques de l'industrie automobile en matière de sécurité, avec la parution, en 1965, de Unsafe at any speed ${ }^{16}$. La révélation que General Motors a fait suivre Nader par un détective privé pour tenter de le discréditer lui vaut une exposition médiatique considérable et légitime sa campagne en faveur de législations plus contraignantes dans de multiples domaines ${ }^{17}$. Fervent partisan de la mobilisation locale et de l'implication civique - également le mode d'action privilégié des organisations écologistes de l'époque - il coordonne des organisations destinées à poursuivre ses travaux, réunies à partir de 1971 sous le nom de Public Citizen. Les causes qu'il embrasse rejoignent en plusieurs occasions celles du mouvement écologiste, en particulier lorsqu'il dénonce en 1974 l'insuffisance des contrôles au sein d'une industrie nucléaire alors en plein essor, cinq ans avant que l'accident de Three Miles Island ne vienne confirmer sa défiance. Son influence sur le Congrès est alors considérable et de nombreuses lois réglementant le commerce, l'industrie ou l'accès à la vie privée doivent beaucoup à son lobbying. Évoquant cet activisme, un critique du New York Times écrit de lui, en 2000, qu'il a eu plus d'influence sur les États-Unis que George W. Bush et $\mathrm{Al}$ Gore réunis depuis le début de leur carrière respective ${ }^{18}$.

Pourtant, son intransigeance et sa propension à attaquer d'anciens alliés, particulièrement après ce qu'il interprète comme une série de trahisons de la part de l'administration Carter, finissent par lasser. Nader quitte la direction de Public Citizen en 1980 et, en dépit d'une constante activité de défense des droits des consommateurs et des citoyens, il disparaît ensuite progressivement du paysage médiatique pendant une quinzaine d'années. Les médias nationaux lui accordent brièvement leur attention en 1988 et 1989 à l'occasion d'une campagne en Californie en faveur d'une loi réglementant les tarifs des assurances automobiles, mais le ton est alors nostalgique, Nader est présenté comme un Don Quichotte en quête d'un «paradis perdu». Le succès de la proposition de loi et les éloges reçus pas Nader pour l'efficacité de sa campagne ne suffisent pas à lui rendre son importance au plan national ${ }^{19}$.

C'est cette popularité résiduelle, ainsi que le souvenir de ses travaux en faveur de lois environnementales dans les années 1970, qui convainquent le Parti vert de Californie de lui demander d'être son candidat pour l'élection présidentielle de $1996^{20}$. Nader, qui avait refusé des offres similaires en 1972 et 1976, déclarant alors qu'il n'entrerait en politique qu'en cas d'invasion martienne, accepte la proposition $^{21}$. Il pose cependant ses conditions : il ne fera pas campagne, ne rejoin-

16. R. Nader, Unsafe at any speed. The designed-in dangers of the American automobile, Grossman Publishers, New York, 1965 [traduction française: Ces voitures qui tuent, Flammarion, Paris, 1966].

17. Vêtu d'une armure de chevalier, par le biais d'un photomontage, il est en couverture de Newsweek en 1968, année où l'écrivain James Ballard l'inclut dans un panthéon pop, aux côtés de Marylin Monroe ou James Dean dans The atrocity exhibition. J. Martin, op. cit., p. 139.

18. A. Clymer, «Green horse candidate», New York Times, 15 octobre 2000.

19. J. Mathews, «California cast vote of confidence in Nader on insurance initiatives», Washington Post, 19 novembre 1998 et M. Fisher, «Ralph Nader's paradise lost», Washington Post, 23 juillet 1989.

20. Selon Brian Tokar, militant écologiste opposé à la candidature Nader, le Parti vert de Californie avait envoyé une lettre à une quarantaine de personnalités leur demandant d'être leur représentant. Seul Nader aurait répondu. B. Tokar, «The Nader for president fiasco», Z Magazine, novembre 1996, <www. zmag.org >.

21. J. Martin, op. cit., p. 226. 
dra pas le parti écologiste, ne suivra pas leur programme et limitera ses dépenses à 5000 dollars, soit moins que le prix d'un spot télévisé de dix secondes à une heure de grande écoute ${ }^{22}$. Soutenu par des activistes écologistes bien au-delà de la Californie, son nom apparaît finalement sur les bulletins de vote dans vingtdeux États sur cinquante. Le système électoral des États-Unis, obstacle principal à la fin du duopole républicain-démocrate selon David Reynolds, impose en effet aux candidats de partis émergents de recueillir dans chaque État un nombre variable de signatures de résidents afin de pouvoir être présent sur les bulletins de vote le jour du scrutin. Ce nombre dépend notamment de l'appartenance ou non à un parti établi, ce qui tend à interdire aux candidats indépendants d'être présents sur l'ensemble du territoire des États-Unis ${ }^{23}$. En 1996, Nader est ainsi candidat sans pouvoir être élu et il recueille un peu moins de 700000 voix, 0,7 \% des votes exprimés ${ }^{24}$. En dépit de son caractère volontairement marginal, cette campagne parvient à susciter un certain intérêt de la part des médias, donnant au mouvement politique écologiste une visibilité nouvelle au plan national. Le pari de la personnalisation semble donc réussi, puisque l'image de Nader permet par la suite au mouvement d'élargir son implantation au-delà des États dans lesquels il avait une base forte ${ }^{25}$.

La candidature de Nader révèle cependant les tensions à l'intérieur de ce mouvement, avec d'une part le GPUSA, favorisant un activisme local dans la tradition des mouvements antérieurs, et d'autre part une nouvelle association coordonnant les efforts des partis locaux à l'échelle fédérale: l'Association des partis verts des États (Association of State Green Parties, ASGP). Le GPUSA ne s'est ainsi rallié qu'au dernier moment à la candidature de Nader, et un membre du conseil national du parti ira jusqu'à parler de fiasco à propos de l'entreprise ${ }^{26}$. C'est donc contre le GPUSA que se constitue l'ASGP, lors d'un rassemblement des organisations écologistes en Virginie, les 16 et 17 novembre 1996, à l'issue des élections ${ }^{27}$. Nader lui-même, lors du rassemblement, enjoint les organisations à s'unir, en refusant implicitement le scepticisme anti-électoral du GPUSA: «Je n’ai jamais compris la soif de pouvoir, les implosions ou les désaccords sur des choses futiles [...]. Tout ce qui vous donne de la visibilité et de l'énergie sans altérer votre ligne politique est bon à prendre ${ }^{28}$.» Ce pragmatisme est au fondement de l'ASGP et pose les bases d'un développement régulier de l'organisation,

22. Ce chiffre de 5000 dollars correspond au seuil à partir duquel un candidat doit publier une liste de ses possessions. S. H. Verhovek, «Unlike '96, Nader runs hard in '00», New York Times, $1^{\text {er }}$ juillet 2000 .

23. En 2004, la Century Foundation, un groupe de réflexion libéral a rédigé un rapport sur les difficultés d'inscription pour un candidat indépendant, en étudiant le cas de la candidature de Nader. Celui-ci avait besoin de 800 signatures seulement pour apparaître sur les bulletins de vote dans le New Jersey, contre 153035 en Californie, un rapport de 1 pour 200. T. A. Wang «Crashing the parties. The problem of ballot access », 2004, <www.tcg.com>.

24. Les résultats d'élections mentionnés proviennent des chiffres officiels, <www.fec.gov>.

25. J. C. Berg, «Greens in the USA», in E. G. Frankland, P. Lucardie et B. Rihoux (dir.), Green parties in transition. The end of grassroots democracy?, Ashgate publishing, Burlington, 2008, p. 249.

26. B. Tokar, op. cit.

27. P. Mazza, «Reinvigorated by Nader campaigns, U.S. Greens gather to plan next steps », minutes de l'ASGP, 1996, <www.gp.org >. En sus de la couverture de la convention, l'article offre la réponse de l'ASGP à l'article de Tokar cité plus haut.

28. R. Nader, cité par Mazza, ibid. 
dont le nombre d'adhérents croîtra d'environ $10 \%$ par an jusqu'en $2004{ }^{29}$. Nader avait d'ailleurs fait de cette construction de l'organisation son objectif avoué en début de campagne ${ }^{30}$, l'inscrivant dans la ligne de ses projets d'action citoyenne concertée poursuivis sous diverses formes depuis les années 1960.

On devine pourtant déjà l'écart entre l'objectif de Nader - remodeler le jeu politique au nom de la démocratie - et celui poursuivi par les écologistes, qui souhaitent utiliser le système électoral comme relais de l'action locale, pour promouvoir un programme précis centré sur l'environnement et la justice sociale. Au-delà de la convergence d'intérêt, la hiérarchie des fins et des moyens est on ne peut plus différente.

\section{Au risque de l'homme présidentiel : l'élection de 2000}

Les élections législatives de mi-mandat, en 1998, confirment la progression de l'ASGP, qui dispose désormais d'un réseau d'élus locaux (soixantedeux, dans quinze États), même s'il réalise des résultats insignifiants dans les élections majeures ${ }^{31}$. En 1998, le New York Times rapporte l'inquiétude des élus démocrates devant la progression du parti, à l'occasion d'un scrutin local au Nouveau-Mexique, et on commence à parler de «green bashing », l'acharnement médiatique contre les verts ${ }^{32}$. L'élection de 2000 prend donc l'allure d'un test pour un mouvement qui se consolide, d'autant plus que l'effondrement du Parti de la réforme de Ross Perot (qui atteignait $19 \%$ des suffrages en 1992, moins de la moitié en 1996), leur permet d'espérer devenir la troisième force nationale. L'enjeu est également financier, puisqu'en atteignant $5 \%$ des suffrages, le parti accéderait aux fonds fédéraux pour préparer les élections suivantes. C'est cet objectif de $5 \%$ que se fixera Nader dès le début de sa campagne, affirmant une nouvelle fois qu'il ne vise pas la présidence, mais la construction d'un parti capable de présenter une alternative à ceux qu'il qualifie de «républicrates ». Crédité d'entre 5 et $10 \%$ dans les sondages au cours de la campagne, il est cette fois présent dans la quasi-totalité des États (quarante-quatre), théoriquement éligible, et reçoit le soutien d'intellectuels comme Howard Zinn et Noam Chomsky, ainsi que celui de nombre de célébrités de l'industrie du divertissement.

La campagne de 2000 exacerbe le schisme de l'élection précédente, entre les «réalistes» de l'ASGP et les «fondamentalistes» du GPUSA, bien que les deux groupes se réclament des dix valeurs fondamentales établies en 1984. En particulier, l'ASGP se rallie au protocole de Kyoto, quand le GPUSA le juge nettement insuffisant ${ }^{33}$. Les documents programmatiques des deux partis prennent

29. S. Greenfield, «The decline of the Green Party», Counterpunch, 19 mars 2005, <www.counterpunch.org/greenfield03192005.html>.

30. Cité dans B. Harden, «In political marriage, Nader dons his own shade of green», Washington Post, 21 mai 1996.

31. M. Feinstein, «1998: record victories, candidates pace Greens,» Green Pages, hiver 1999, $<$ greenpages. wordpress. com/?s = 1998>.

32. Ibid. et J. Brooke, «Green Party grows (so does Democrats'dismay)», New York Times, 30 juillet 1998.

33. Pour une analyse plus précise des divergences entre les deux organisations écologiques sur ce sujet du réchauffement climatique, ainsi que sur la position du gouvernement fédéral vis-à-vis du protocole de Kyoto, voir J. Kovel «Global warming and realo-fundi Greens. What's being done to transform the sys- 
d'ailleurs soin en 2000 de les distinguer nettement l'un de l'autre, même si le discours public se focalise sur le «Green Party», sans autre précision. L'ASGP et le GPUSA incarnent les deux termes de l'alternative identifiée par David Reynolds : séduire la base ou chercher une majorité ${ }^{34}$, deux objectifs rendus incompatibles par le système électoral majoritaire des États-Unis. Le développement de l'ASGP aux dépens du GPUSA est une victoire pour une approche pragmatique du jeu électoral mais aussi des enjeux écologiques.

Nader, qui se réclame lui aussi du «Green Party», est bien le candidat de l'ASGP, dont il accepte le programme et utilise l'organisation mise en place après 1996. Ce programme se place d'emblée sur une position de compromis, dans laquelle l'écologie est remplacée par une approche environnementale réformiste plutôt que radicale. Il comporte quatre grandes sections: démocratie, justice sociale et égalité des chances, gestion responsable de l'environnement, et économie responsable (economic sustainability, de la mise en place de lois antitrust au développement rural) ${ }^{35}$. Dans ce document, la question de la réforme électorale et de la démocratie constitue la toute première section, la plus lisible, en quatre points bien délimités. Articulée à l'idée de justice sociale, c'est elle qui domine le document, dont les questions environnementales ne représentent qu'un quart environ. Par ailleurs, le mot «écologie», renvoyant à une conception globale, n'apparaît dans les autres parties du texte qu'en deux occasions: pour affirmer la nécessité d'une coopération internationale (section I) et pour souligner la compatibilité entre écologie et économie responsable (introduction de la section III). Certes, le mot «environment» est plus courant dans le discours politique nordaméricain, mais a contrario, c'est bien d'écologie dont il est question à une trentaine de reprises dans le programme du GPUSA, un document plus court publié la même année ${ }^{36}$. La lecture des mesures proposées en faveur de l'environnement révèle surtout des déclarations d'intention (par exemple, la troisième proposition dans la rubrique «politique de transport»: «Nous devons développer le réseau ferroviaire du pays, les trains de passagers à grande vitesse ainsi que les systèmes ferroviaires urbains légers ») et des propositions ciblées, comme l'établissement de meilleures normes en matière de consommation automobile (une promesse non tenue de l'administration Clinton-Gore). Sur les points pour lesquels une option radicale est évoquée - comme l'abandon progressif des combustibles fossiles, le basculement vers les énergies renouvelables - ni calendrier ni objectif contraignant ne sont proposés. À l'inverse, des mesures de portée moindre sont détaillées et accompagnées de plans d'action complets. Là encore, le document se place donc dans la continuité de ce qu'avait entrepris Nader trente ans auparavant: la mise en place de réglementation contraignante sur des points précis, mais sans remise en cause du système. Pourtant, il s'en éloignera encore lors de la campagne.

tem ?», Z magazine, février 1997, <www.zmag.org/zmag/feb01kovel.html>. Je lui emprunte les termes de l'opposition entre écologie «fondamentaliste» et «réaliste».

34. D. Reynolds, op. cit., p. 254.

35. Green Party Plaform 2000, site de du GPUS (ex-ASGP): <www.gp.org >.

36. Platform of the Greens/Green Party USA, site du GPUSA : <www.greenparty.org>. 
«Environnement» et «démocratie», sont les mots clés de ses deux allocutions devant l'ASGP en 2000, pour proposer sa candidature puis pour accepter sa nomination en tant que candidat du parti, comme l'illustre le passage suivant, tiré de l'introduction à son discours de candidature:

«Aujourd'hui, notre société fait face à de graves problèmes dans les domaines de la santé, de l'éducation, de l'organisation du travail, de l'énergie et de l'environnement. Ce sont des problèmes pour lesquels les citoyens actifs ont des solutions, mais leurs voix ne parviennent pas à franchir la faille dans le système démocratique [democracy gap]. Les groupes citoyens et les intellectuels individuels produisent une somme prodigieuse d'idées, d'informations et de solutions, tandis que notre gouvernement nous est dérobé par le pouvoir des multinationales ${ }^{37}$.»

Réformes du système politique et questions environnementales sont ici hiérarchisées: les mesures en faveur de l'environnement sont conditionnées par une question plus vaste, celle du dysfonctionnement systémique de la démocratie américaine qui découle de la soumission de la politique au pouvoir économique. Sans nier les problématiques soulevées par les écologistes, Nader les subordonne donc à la question de la représentation, son propre cheval de bataille. Si l'articulation entre action locale et pouvoir politique est un problème à résoudre pour les écologistes, elle apparaît comme un enjeu de campagne en elle-même pour Nader.

Lorsqu'il développe sa position sur ces questions écologiques, il le fait encore en réformiste plus qu'en idéaliste, et en revenant à la question de la légitimité du pouvoir et à celle de la responsabilité, individuelle ou collective. Concernant le réchauffement climatique ou le trou de la couche d'ozone, mentionnés une seule fois et brièvement au cours des deux discours, c'est ainsi vers les multinationales qu'il se tourne, suggérant de leur faire porter le poids économique des dommages qu'elles causent : "Que dire à propos de la dévastation de l'environnement? Elle n'apparaît pas dans les comptes d'Exxon, de DuPont, de General Motors ou de Peabody Coal. [...] General Electrics n'a pas été jugé comme responsable de l'empoisonnement au PCB de l'Hudson River et n'a reçu qu'une condamnation dérisoire pour ce qu'ils ont fait à la Housatonic River, dans ma région ${ }^{38}$.» La question écologique est ici inscrite dans une perspective résolument anthropocentrique, économique et localisée, où il est question de pollution, de responsabilité privée, plutôt que de dérèglement planétaire, loin d'un modèle biocentrique, qui impliquerait une remise en cause du modèle global de développement ${ }^{39}$. La référence de Nader, c'est le lobbying citoyen du début des années 1970, qui allait mener à l'institutionnalisation de l'écologie et qu'il cite directement en exemple dans sa proposition de candidature. La pollution n'est ici qu'un aspect de la dégradation du rapport de force entre les individus et les grandes entreprises.

37. R. Nader, «Statement announcing his candidacy for the Green Party's nomination for President», 21 février 2000, <www.votenader.com>, via The Internet Archive Wayback Machine, <www.archive. org $>$.

38. R. Nader, «Acceptance statement for the association of state Green Parties nomination for President of the United States », 25 juin 2000, <www.votenader.com>, via The Internet Archive Wayback Machine, <www.archive.org >.

39. F. Duban, «L'institutionnalisation de l'environnementalisme aux États-Unis remise en cause par l'écologisme», Revue française d'études américaines, n 70, p. 45-47. 
Il ne s'agit pas là pour Nader d'une posture de circonstance, mais de la poursuite de son propre mode d'action dans les quarante années précédentes, sur lequel repose encore sa popularité en 2000: initiatives individuelles, formation d'associations citoyennes, lobbying ou action judiciaire collective. Un militant antinucléaire ayant travaillé avec lui dans les années 1970 résume cette position, en notant qu'«il a toujours été plus intéressé par la question du pouvoir des grandes entreprises que par des concepts vagues comme l'environnementalisme, le sexisme ou le racisme ${ }^{40} »$. Si ses engagements rejoignent parfois ceux des écologistes, en particulier dans l'opposition au nucléaire, Nader est en réalité un progressiste, dans la continuité de ce vaste mouvement de réforme du début du $\mathrm{Xx}^{\mathrm{e}}$ siècle, qu'allaient notamment incarner les deux présidents Roosevelt; une tradition dans laquelle la question de la redistribution du pouvoir est cruciale, mais qui refuse de renoncer au système ${ }^{41}$. Cette ère progressiste est aussi celle durant laquelle ont été établies les premières règles de préservation de la nature aux États-Unis ${ }^{42}$ : les convergences sont réelles avec une certaine idée de l'écologie, illustrée par le programme de l'ASGP en 2000, mais les priorités en sont différentes. Celle de Nader est la constitution d'une force capable de déverrouiller le système politique, posée en préalable à la résolution de toutes les autres questions ${ }^{43}$.

Lorsqu'il ne s'adresse pas directement aux écologistes, Nader omet parfois de mentionner toute question environnementale ou les relègue à la périphérie d'un discours focalisé sur l'objectif dominant: la mise en place d'une alternative crédible et citoyenne au bipartisme ${ }^{44}$. C'est particulièrement évident lorsque, peu de temps après sa désignation par l'ASGP, il s'adresse à la NAACP (National Asssociation for the Advancement of Colored People), organisation sensible aux arguments de justice sociale et de réorganisation de la démocratie. Lorsque l'environnement est mentionné, c'est en quelques mots, sous une forme désormais familière, qui identifie les problèmes écologiques aux grandes entreprises : «Qui s'est opposé au mouvement vers un environnement sain dans ce pays? [...] Qui s'y est opposé? Les multinationales ${ }^{45}$.» Lorsque Nader détaille le programme sur lequel il s'engage («Let me go through the agenda»), son quasi-silence sur la question environnementale est flagrant. Fin du financement privé lors des élections, adoption d'une législation antilobbys, couverture maladie universelle, fin des subventions aux entreprises, renforcement du contrôle public des médias, réforme de l'éducation et un «plan Marshall» pour abolir la pauvreté aux États-

40. M. Mariotte, interviewé et cité par J. Martin, op. cit., p. 173.

41. J. Izbicki, «Progressive», in A. Bullock et S. Trombley, The Norton dictionary of modern thought, W. N. Norton and Cie, New York \& Londres, 1999, p. 691.

42. À l'exception de la fondation de Yellowstone en 1872, qui précède de peu cette ère réformiste.

43. S. H. Verhovek, op. cit.

44. Il faut bien entendu être prudent en rapportant ce qui n'est pas dans les interviews, puisque celles-ci sont l'objet d'un inévitable processus éditorial. Néanmoins, qu'il s'agisse d'une lettre au New York Times dans laquelle Nader justifie sa campagne («Why I'm running. The Nader critique», New York Times, 4 juillet 2000) ou d'une interview pour le San Francisco Chronicle («It's not easy being Green», San Francisco Chronicle, 24 août 2000), il est aisé de recenser des textes ne comportant aucune mention des questions environnementales, au cours de cette campagne.

45. R. Nader, «We live in an appartheid society - Speech to the NAACP convention», Z Magazine, 11 juillet 2000, <www.zmag.org/naacpnader.htm>. 
Unis: l'ensemble de ces points appartient bien au programme défini par l'ASGP, empruntant des mesures à trois de ses quatre sections, mais Nader omet entièrement le chapitre environnemental. Il s'agit bien d'un positionnement radical par rapport à la position démocrate sur ces différents points, mais cette radicalité s'exprime dans le champ social et politique plutôt qu'écologique.

Ce discours devant le NAACP, sans intervention éditoriale extérieure, montre que la discrétion de la question écologique au cours de la campagne n'est pas due aux choix opérés par les médias relayant les interventions du candidat. C'est bien Nader lui-même qui s'abstient fréquemment d'aborder le sujet, dès lors qu'il ne s'adresse pas à une audience composée de militants écologistes. Il n'omet en revanche jamais de rappeler à chaque intervention qu'il est le candidat du Parti vert, et c'est finalement cette mention du nom du parti qui lui tient lieu de position sur l'environnement. En intégrant un homme providentiel proche de leurs idées sur bien des points mais à la philosophie sous-jacente différente, l'ASGP s'est doté d'une personnalité plus forte médiatiquement que le parti lui-même, mais prêchant résolument à la majorité plus qu'à la base. Cette fracture entre les Verts et leur candidat ne date pas de 2000, et certains commentateurs écologistes l'avaient soulignée à l'issue de sa première campagne présidentielle, notant avec acidité: "Il est difficile de venir à bout du réchauffement climatique avec un procès $^{46}$.» De fait, si le progressisme se marie avec l'idée de «conservation», il offre un cadre intellectuel dans lequel l'humain prime, rendant difficile l'abord de questions à long terme et à l'échelle globale.

Pour l'ASGP, le pragmatisme de leur candidat produit des résultats paradoxaux. L'exposition reçue par le parti est d'autant plus grande que Nader adopte un discours rassemblant à gauche et fédérant au-delà des cercles écologistes. Bien qu'il ait affirmé son peu de goût pour la personnalisation de la politique, sa campagne est médiatisée et perçue comme reposant sur sa personnalité, sa popularité passée et par extension ses convictions, bien plus que sur le programme défini par son parti. L'ASGP est alors réduit au statut de «parti de $\operatorname{Nader}^{47}$ », en dépit des efforts de ses adhérents pour redresser cette image. Cette approche pose également un problème de cohérence interne de la campagne, puisque, paradoxalement, Nader appelle à voter pour un candidat n'ayant aucune chance d'être élu: lui-même. De fait, aucune des trois organisations environnementalistes qui s'expriment au sujet de l'élection, Friends of the Earth, le Sierra Club et la League of Conservation Voters, ne choisira de soutenir Nader, précisément au nom du pragmatisme: «Nous faisons face à la réalité. Aux États-Unis, le candidat du troisième parti n'est pas élu président ${ }^{48}$.» Ce choix indique à la fois les limites de la campagne de Nader, mais aussi celles d'une écologie politique indépendante, puisque le Sierra Club compte à lui seul 600000 membres à l'épo-

46. . J. Kovel, op. cit.

47. J. Dao, «History could be Green Party's toughest opponent», New York Times, 2 novembre 2000.

48. J. Dao, «Friends of the Earth decides to back Gore, reluctantly», New York Times, 5 septembre 2000 . 
que de cette élection, un nombre nettement plus élevé que les 250000 adhérents de l'ASGP 49 .

L'argument du réalisme politique explique à son tour en grande partie le score final décevant obtenu par Nader : 2,8 millions de votes, soit 2,7 \% de l'électorat. Beaucoup plus qu'en 1996, mais loin des $5 \%$ nécessaires pour obtenir un accès aux fonds fédéraux et surtout nettement moins que ce que les sondages avaient annoncé. Bien qu'il soit parvenu à attirer de nouveaux électeurs, Nader n'a pas su créer de mouvement d'ampleur qui aurait traduit le fort soutien manifesté pendant sa campagne ${ }^{50}$. Le résultat le plus marquant de cette élection, celui qui marque l'échec de la stratégie visant à conquérir une majorité plutôt que de renforcer la base est ce chiffre frappant: parmi les électeurs déclarant que Nader était leur candidat préféré, seul un cinquième a voté pour lui. Un autre cinquième de ses partisans a choisi George $\mathrm{W}$. Bush et trois cinquièmes ont opté pour $\mathrm{Al}$ Gore. En d'autres termes, Nader a séduit, mais son argument principal, la nécessité de construire un troisième parti, est précisément celui que les électeurs ont rejeté par ce vote «stratégique ${ }^{51} »$ : sa défaite est une victoire en creux du système bipartite. Pour l'ASGP, elle signifie aussi l'échec de ce pari de l'homme présidentiel et de la transformation du mouvement écologiste en parti efficace à l'échelle fédérale. L'échec stratégique est redoublé d'une part par les divisions internes du mouvement et de l'autre par les accusations portées contre Nader - et, par extension, contre les écologistes - d'avoir «gâché» l'élection d'Al Gore.

Dans ses derniers mois de campagne, alors que les sondages tardaient à annoncer la traditionnelle chute des intentions de vote des candidats minoritaires $^{52}$, sa campagne avait rencontré une opposition de plus en plus marquée de la part des démocrates ${ }^{53}$. Si Ross Perot avait contribué à faire élire puis réélire Bill Clinton, Nader menace quant à lui les démocrates par leur gauche. La situation en Floride confirmera ces craintes, puisqu'après recomptes et intervention de la Cour suprême, l'écart officiel en faveur de Bush sera de 537 voix, quand Nader en a obtenu 97488 . L'idée selon laquelle Nader aurait permis l'élection de Bush est encore un sujet de débats partisans intenses; elle demande à être détaillée. En effet, outre Nader, six autres «petits» candidats ont obtenu un nombre de voix supérieur à l'écart entre Gore et Bush. Il est impossible de déterminer avec certitude si la présence de Nader a été le facteur déterminant de cette élection, mais cette controverse constitue désormais le point saillant dans tous les bilans de cette campagne de $2000^{54}$.

49. M. Schreuers, op. cit., p. 212 et E. Nieves, «This time, Nader promises, it's a serious run for President», New York Times, 7 mars 2000.

50. B. C. Burden, «Minor parties in the 2000 presidential election», in H. F. Weisberg et C. Wilcox, Models of voting in presidential elections. The 2000 U.S. election, Stanford Univ. Press, Stanford, 2004, p. 218.

51. Ibid., p. 210-211.

52. Ibid., p. 212-214.

53. Le New York Times fut également accusé, non sans raison, d'avoir consacré une part importante de ses éditoriaux à attaquer la légitimité de la candidature de Nader.

54. Nader lui-même est convaincu que Gore a perdu l'élection sans que sa candidature en soit la cause. R. Nader, «Dear Conservatives upset with the policies of the Bush Administration», site Vote Nader 2004, <www.votenader.org/why_ralph/index.php? cid = 14>. 
Du point de vue de Nader, l'exposition reçue durant la campagne constitue une victoire pour l'ASGP, à qui il prédit à l'issue de l'élection qu'il deviendrait un parti majeur en une douzaine d'années ${ }^{55}$. De fait, le parti continue de se renforcer, mais s'engage rapidement dans une nouvelle phase du débat entre pragmatisme et idéalisme. Il s'agit cette fois de savoir si les écologistes peuvent se permettre de risquer d'offrir la victoire aux républicains dans des élections majeures ou s'il faut considérer que les démocrates se condamnent eux-mêmes en adoptant un discours trop consensuel. La question est centrale lors de la convention de mi-mandat en 2002, et elle le sera plus encore lors du choix du candidat pour l'élection présidentielle en 2004. Nader fait alors savoir qu'il sollicite le soutien du parti mais ne souhaite pas en être le candidat officiel, privilégiant une alliance plus vaste. Il choisit également de ne pas se rendre en personne à la convention nationale désignant le candidat de l'ASGP (devenu entre-temps le Green Party of the United States, GPUS). Une part importante du parti se dit alors convaincue qu'une nouvelle candidature de Nader obligerait le parti à se justifier du résultat de l'élection précédente, rendant illisible leurs engagements ${ }^{56}$. Le 26 juin 2004, les militants rassemblés optent pour David Cobb, candidat ayant promis de ne pas livrer bataille à John Kerry dans les États disputés. Il recueillera 0,1\%, des voix. Nader, de son côté, se présentera avec l'appui du Parti de la réforme, atteignant au total 0,4\% des suffrages (moins que lors de sa campagne virtuelle de 1996) dans une élection qui marque la fin de presque huit ans d'une coopération lâche entre l'avocat des consommateurs et l'écologie politique.

\section{Bilan}

Le choix de la personnalisation n'a pas été que négatif pour l'écologie politique américaine: les réseaux et la logistique mis en place pour soutenir les candidatures de Ralph Nader, ainsi que ses interventions sur le terrain dans les élections intermédiaires de 1998, ont permis à l'ASGP de se structurer et d'acquérir une visibilité qu'un porte-parole plus proche de la ligne du parti, mais moins médiatique, n'aurait sans doute pas suffi à susciter. L'implantation locale des Verts est solide, avec plus de 260000 membres en 2008, un peu moins qu'à leur apogée en 2004, mais nettement plus que les 118000 de $1998^{57}$. Le nombre d'élus locaux n'a également pas cessé de progresser, dépassant les 200 en $2007^{58}$. A contrario, les scores médiocres obtenus par Cobb en 2004, puis par Cynthia McKinney, ex-démocrate, en 2008 (0,12 \% du vote populaire), permettent de mesurer la difficulté persistante pour un petit parti de se faire entendre à l'échelle fédérale. Le relatif succès de l'expérience Nader ne s'est donc pas traduit par le déblocage des obstacles à l'émergence d'un troisième parti et c'est au contraire à un recentrement vers l'échelle locale d'origine que l'on semble assister, tant la

55. A. Clymer «Nader sees green building status as a major party», New York Times, 18 novembre 2000 .

56. M. L. Sifry, «Ralph redux ?», The Nation, 24 novembre 2003.

57. J. C. Berg, op. cit., p. 252 et Ballot Access News, mars 2008, <www.ballot-access.org>.

58. D. Arthur, «How did we get here? A brief history of the Green Party around the world», Green Pages, été 2007, <greenpages. wordpress. com/?s = Nader + $2000+$ Arthur $>$. 
campagne menée en 2008 a été minimaliste. On peut même penser que le vote «stratégique », déjà crucial en 2000, ne peut que se renforcer à l'avenir, au vu du précédent, réel ou supposé, de l'élection de George W. Bush. Au-delà de cette coïncidence historique, ce que prouvent ces années d'association entre Nader et l'écologie politique, c'est bien le rôle déterminant du facteur personnel dans le système électoral des États-Unis. Sur son seul nom et sans faire campagne, en 1996, Nader avait en effet rassemblé cinq fois plus de voix que Cynthia McKinney douze ans plus tard, en dépit de la consolidation du parti dans l'intervalle. L'implantation à l'échelle fédérale d'un parti écologique ne semble pouvoir se passer de l'émergence d'une personnalité forte, que ne décourageraient par les obstacles systémiques auquel l'organisation est confrontée.

En ce qui concerne Ralph Nader, ses positions sur les questions environnementales restent d'une grande constance depuis le début de son engagement dans le débat public, et s'il a changé l'écologie politique aux États-Unis, celle-ci ne l'a que peu influencé en retour. En dépit de ses convictions environnementalistes, il n’a jamais été un écologiste. Il n'a jamais adhéré à l'ASGP, a cherché à maintenir son indépendance jusque dans l'organisation de ses campagnes successives. Le résultat de 2000 est avant tout le sien, pour le meilleur et pour le pire, et s'il est sorti de l'éclipse médiatique des années 1990, son image immaculée a été considérablement ternie par son engagement politique, dans lequel il semble de plus en plus seul : présent sur les bulletins de vote de quarante-cinq États en 2008, il a obtenu $0,56 \%$ du vote populaire. Le contraste avec la victoire de Barack Obama lors de cette élection est édifiant, tant les parallèles entre les deux hommes sont nombreux. Mettant en avant les questions écologiques (en particulier par le mécanisme du «cap and trade», système de plafonnement et d'échange des émissions de gaz à effet de serre) et légitimé lui aussi par une indéniable expérience d'activisme local, Obama a su incarner par son parcours, sa stratégie de campagne et sa couleur de peau un idéal de renouveau de la vie politique, mais a construit sa victoire en s'appuyant sur l'appareil d'un des deux grands partis. À l'inverse, Nader ne semble plus, à 73 ans et isolé de ses anciens alliés, en mesure d'être de nouveau l'homme providentiel du progressisme ou de l'écologie. 
